Case Report

\title{
SOME PARASITOLOGICAL, PATHOLOGICAL AND IMMUNOHISTOCHEMICAL EXAMINATIONS IN SHEEP NATURALLY INFECTED BY TRICHURIS OVIS
}

\author{
P. T. Iliev ${ }^{1 *}$, A. Ivanov ${ }^{1}$, Z. Kirkova ${ }^{1}$, K. Hristov ${ }^{2}$, K. Dinkova ${ }^{3}$, J. Ananiev ${ }^{3}$ \\ ${ }^{1}$ Department of Veterinary Microbiology, Infectious and Parasitic Diseases, Faculty of Veterinary \\ Medicine, Trakia University, Stara Zagora, Bulgaria \\ ${ }^{2}$ Department of Obstetrics, Gynecology, Biotechnology of Reproduction, Pathological Anatomy and \\ Biochemistry, Faculty of Veterinary Medicine, University of Forestry, Sofia, Bulgaria \\ ${ }^{3}$ Department of General and Clinical Pathology, Medical Faculty, Trakia University, Stara Zagora, \\ Bulgaria
}

\begin{abstract}
A case of Trichuris ovis infection in a lamb is presented. In June 2012 a herd of 12 infected with T. ovis lambs in Brestnik (Southern Bulgaria) were discovered. A diarrhea and loss in body weight in 2 lambs have been observed. Despite antibiotic treatment one of the animals died. Parasitological examination was done. During necropsy of the gastro-intestinal tract, adult worms in the caecum were found. Standart staining and immunohistochemistry were performed to detect the cell population in the affected areas of the caecum.
\end{abstract}

Key words: Trichuris ovis, immunohistochemistry, sheep

\section{INTRODUCTION}

Trichuris ovis (Adenophorea: Trichuridae) is a caecal parasite most prevalent in ruminants irrespective of age, gender and breed of the hosts (1). It is usually detected incidentally during routine diagnostic or necropsy. The heavy infections are rare but may be seen in very young lambs (2). Generally, natural infections are seldom severe enough to cause clinical disease (3). Due to its thin, thread-like anterior end of the body, T. ovis is more commonly known as ovine whipworm. This parasite is soil-transmitted helminth with direct life cycle (4). Eggs contain an unsegmented embrio when they laid. Larvae are formed and become infective within the shell of the eggs after disposing them into the environment. The hosts become infected orally by ingesting food, contaminated with eggs. Having reached in small intestine by gastric passage, the eggs hatch and the released larvae pass to the caecum. There, they burrow into the mucosal layer and turn into adult worms. After reaching sexual maturity, the worms remain deeply

\footnotetext{
*Correspondence to: Petar T. Iliev, Department of Veterinary Microbiology, Infectious and Parasitic diseases, Faculty of Veterinary Medicine, Trakia University, 6000 Stara Zagora, e-mail: petyo_todorow@abv.bg
}

embedded in the intestinal mucosa by their anterior end $(3,4,5)$. The penetration of $T$. ovis and T. scrjabini into the mucosa causes not only mechanical damage but also histological changes corresponding to the degree of infection. The main changes consist of a local lymphocyte infiltration, mucosal desquamation, erosions in the place of attachment and hypersecretion of the intestinal glands (6).

The information about the histological disorders during $T$. ovis infection in the caecum is limited. In contrast, many studies of the inflammatory cell infiltration in T. vulpis, T. suis and T. muris infection have been done. Here, we describe and compare the immunohistology and histopathology of the caecum infested by sheep whipworm $T$. ovis, using canine, porcine and murine whipworms as comparative models.

\section{CASE HISTORY}

In June 2012 the owner of herd of 12 lambs observed that in 2 of the animals diarrhea and faintness were manifested with short periods of improvement of the general condition. Satisfactory outcome after treatment with Tylovet ${ }^{\circledR}$ has not been observed and one of the 
animals died. The carcass was deposited at Trakia University for necropsy.

The partial necropsy and parasitological observations were made in Veterinary Faculty, Department of Parasitology, and immunohistochemistry - in Medical Faculty, Department of General and Clinical Pathology, Trakia University.

\section{MATERIALS AND METHODS Parasitological examinations}

- Flotation technique

Eleven individual fecal samples were examined by standard saline flotation technique.

\section{- Species identification}

For this task we used some morphological and morphometrical characteristics of adult whipworms such as total body length, length of the anterior esophageal section (LES), length of the posterior section (LPS), ratio between the LES and LPS, collocation and shape of the vulva, length and shape of the spicules, all described by Soulsby (3), Charakchiev (7) and Kuchai et al. (1).

\section{Pathological examination}

The necropsy was made by routine protocol and only the gastro-intestinal tract was examined.

\section{Staining studies}

- Standard staining

For standard staining we used hematoxylin and eosin stain (H\&E stain) on the paraffin sections with $5 \mu \mathrm{m}$ thick.

\section{- Immunohistochemisry}

For immunohistochemical staining, the paraffin blocks were prepared using caecal tissues. Paraffin sections $5 \mu \mathrm{m}$ thick were dewaxed in two xyllenes and were rehydrated in ethanol. Later, they were washed in $0.1 \mathrm{M}$
ILIEV P.T., et al. phosphate buffered saline (PBS), pH 7.4, incubated in $1.2 \%$ hydrogen peroxide in methanol for $30 \mathrm{~min}$, and rinsed in $0.1 \mathrm{M} \mathrm{PBS}$, $\mathrm{pH} \mathrm{7.4,} \mathrm{for} 15 \mathrm{~min}$. Immunohistochemical reaction were carried out using Monoclonal Mouse Anti-Human CD4, Clone 4B12 (DAKO), Monoclonal Mouse Anti-Human CD68, Clone KP1 (DAKO) and Monoclonal Mouse Anti-Human Granzyme B, Clone GrB7 (DAKO). After washing three times in PBS, the slides were incubated with DAKOREAL $^{\mathrm{TM}}$ En-Vision $^{\mathrm{TM}}$ detection system (DAKO) for $30 \mathrm{~min}$, then visualized with 3,3'diaminobenzidine and counterstained with hematoxylin. We used a known tissue to exhibit high levels of marker for positive control and for negative control, the primary antibody was replaced with PBS.

\section{RESULTS}

\section{Parasitological findings}

Coprological examination showed the presence of Trichuris spp. eggs in all examinated animals. Species identification of 13 female and 7 male adult parasites confirmed the monoinfection by $T$. ovis.

\section{Pathological findings}

Macroscopically the mucosa of the caecum was oedematous and some petechial hemorrhages were presented. We found plenty of mucus and worms deeply embedded in the caecal mucosa. No necroses were discovered.

Histologically, hematoxylin and eosin stained sections revealed the parasite body surrounded by numerous macrophages, lymphocytes, some neutrophils, and single eosinophilic leukocytes (Figures 1 and 2 a, b). Mucosa of the caecum underwent intense inflammatory infiltration accompanied by rising in the number of lymphocytes and neutrophils.

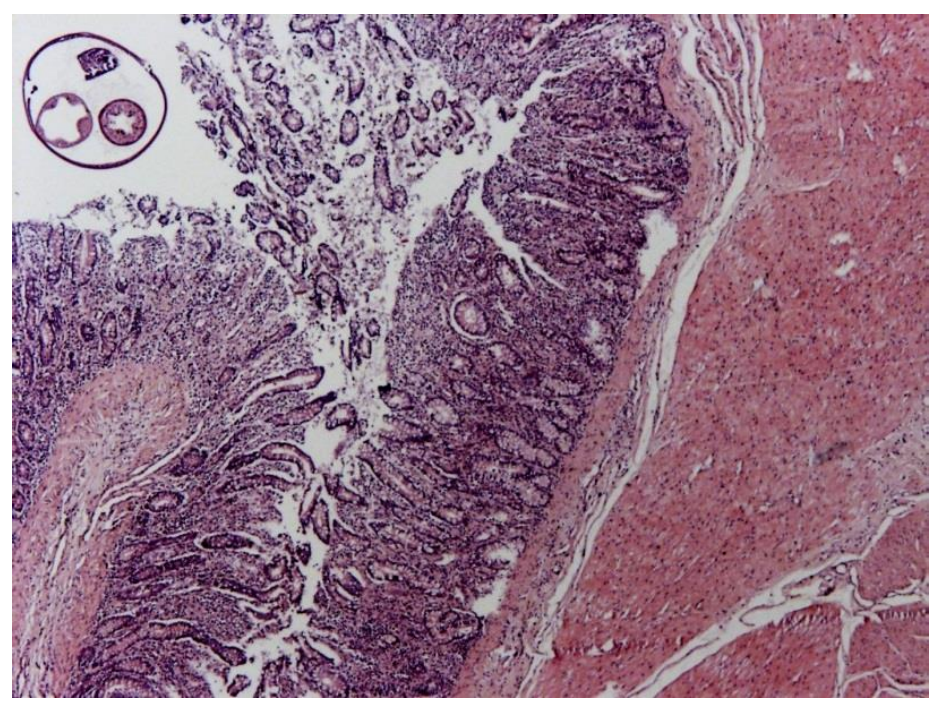

Figure 1. Caecum. Anterior thin end of T. ovis surrounded by dystrophic mucosal layer. H/E, 200X 


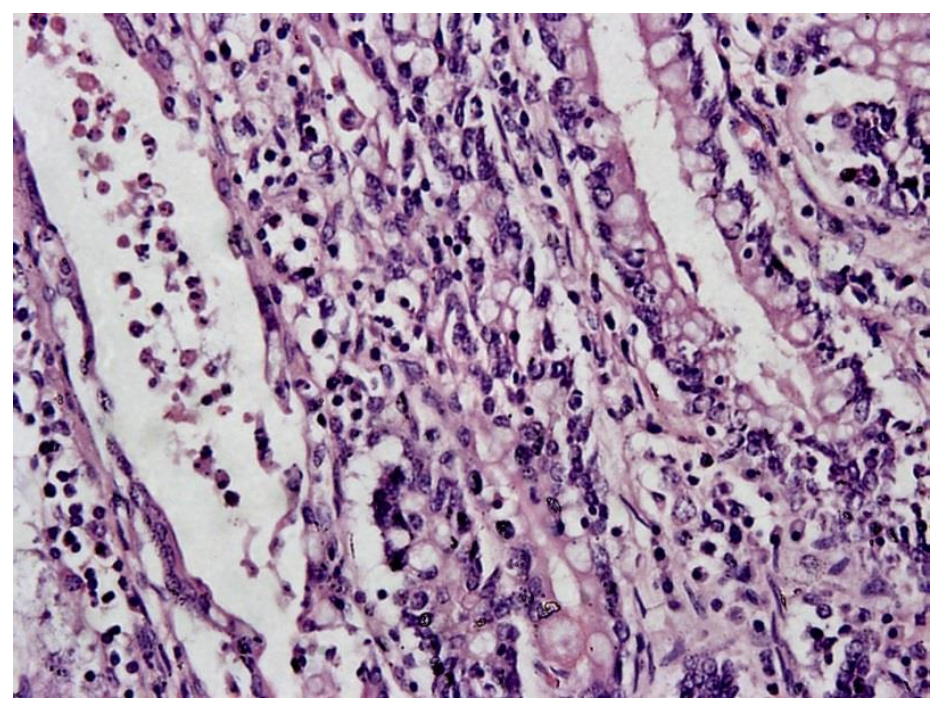

ILIEV P.T., et al.

Figure 2a. Caecum. Inflammatory cell infiltration consisting of macrophages, lymphocytes and a number of neutrophilic leukocytes. H/E, 400X

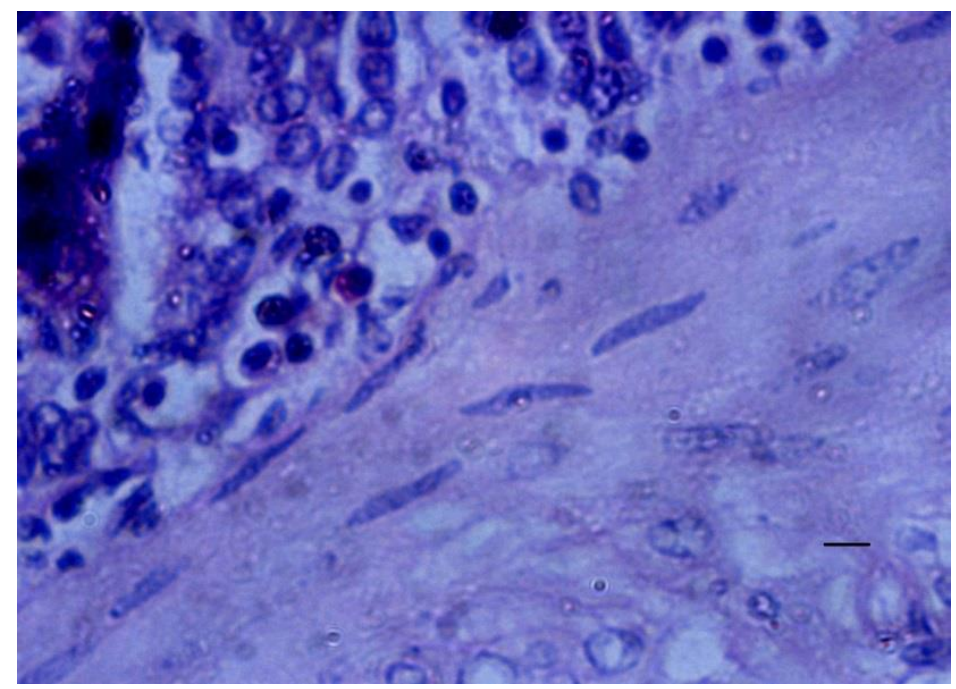

Figure 2b. Caecum. Mucosal fibroblast cells and eosinophil infiltration. H/E, Bar $=25 \mu \mathrm{m}$.

Immunohistochemistry investigation

Immunohistochemical studies confirmed strong staining for CD68+cells in caecal mucosa (Figure 3). CD4+ cells were also occasionally observed in this region (Figure 4). There are no NK positive cells (Granzime B negative) in investigated tissue.

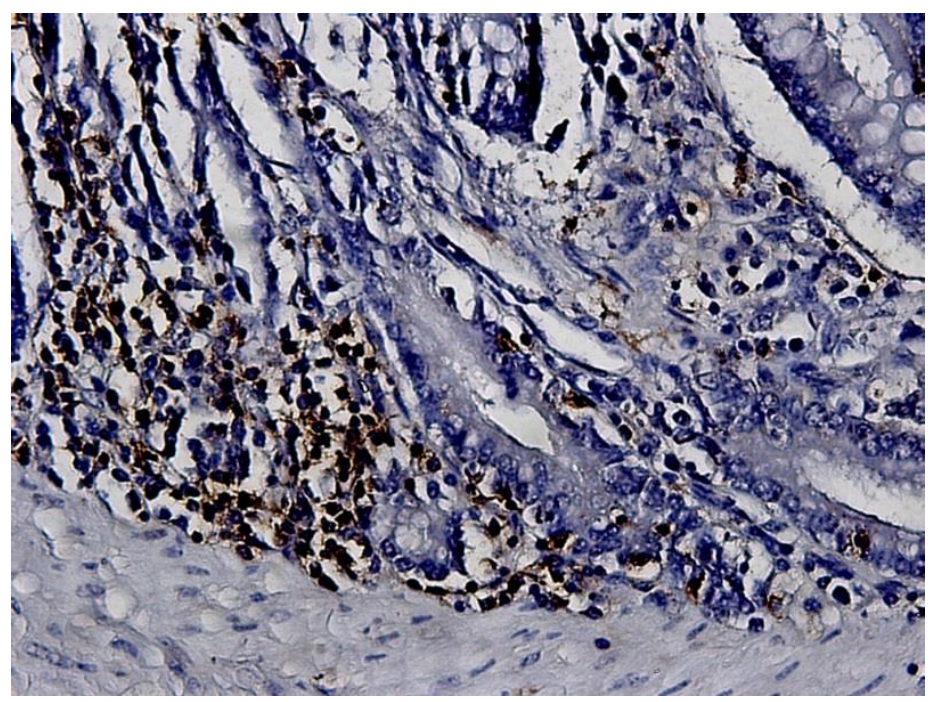

Figure 3. Caecum. Presence of intraepithelial CD68+ cells in the mucosa. Immunohistochemical staining, 200X 


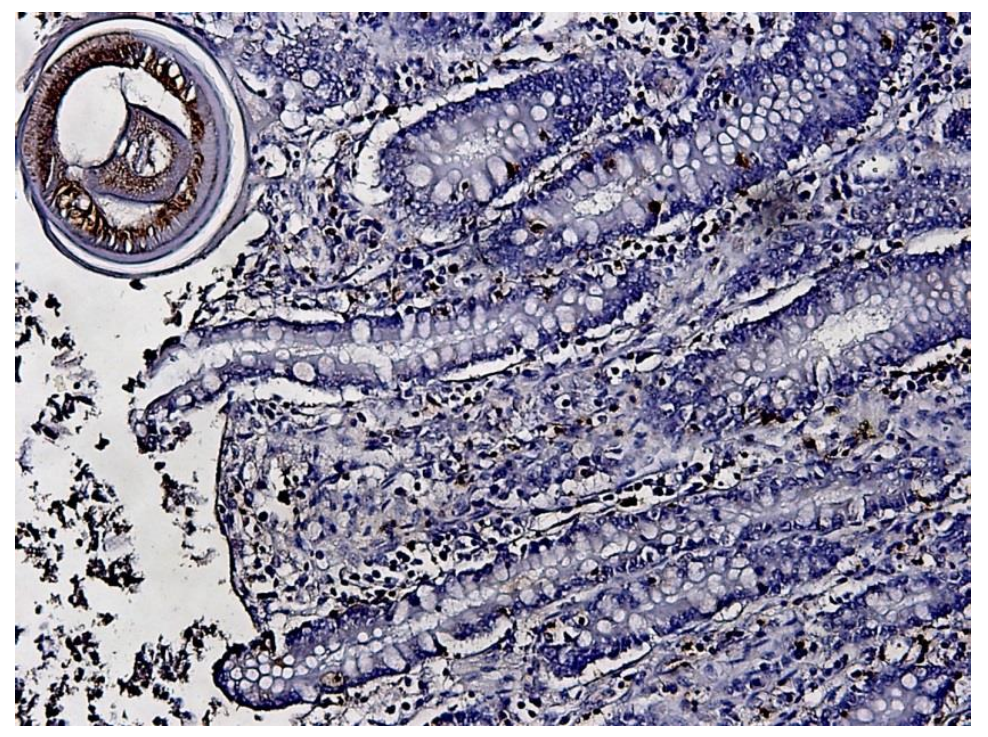

ILIEV P.T., et al.

Figure 4. Caecum. Presence of CD4+ cells in the mucosa. Immunohistochemical staining, 200X

\section{DISCUSSION}

It is known that the adult trichurids parasitize deeply embedded in bowel. According to Kirkova et al. (8) both mature parasites and larvae are able to cause mechanical changes in the intestinal mucosa. In contrast, Efremov and Shihobalova (see 6) report that T. muris infection does not induce any macroscopic changes in the cecum. Our results were similar to those of Bratanov et al. (6). The authors found that the degree of infection with ovine whipworms is able to cause from slight changes as petechial hemorrhages to better expressed hemorrhages of the bowel. Similar changes were observed in $T$. suis infection in pigs (9). In our case a moderate infection in the dead lamb was discovered. A diarrhea was found only in 2 of 12 infected animals. Given the weak pathological changes in the lamb we assume that the cause of death was not due to whipworm infection. Likely, a secondary microflora has been implicated. According to Smith et al. (10) Trichuris spp. do not play a significant pathogenic role, especially in ruminants which is in agreement with our findings.

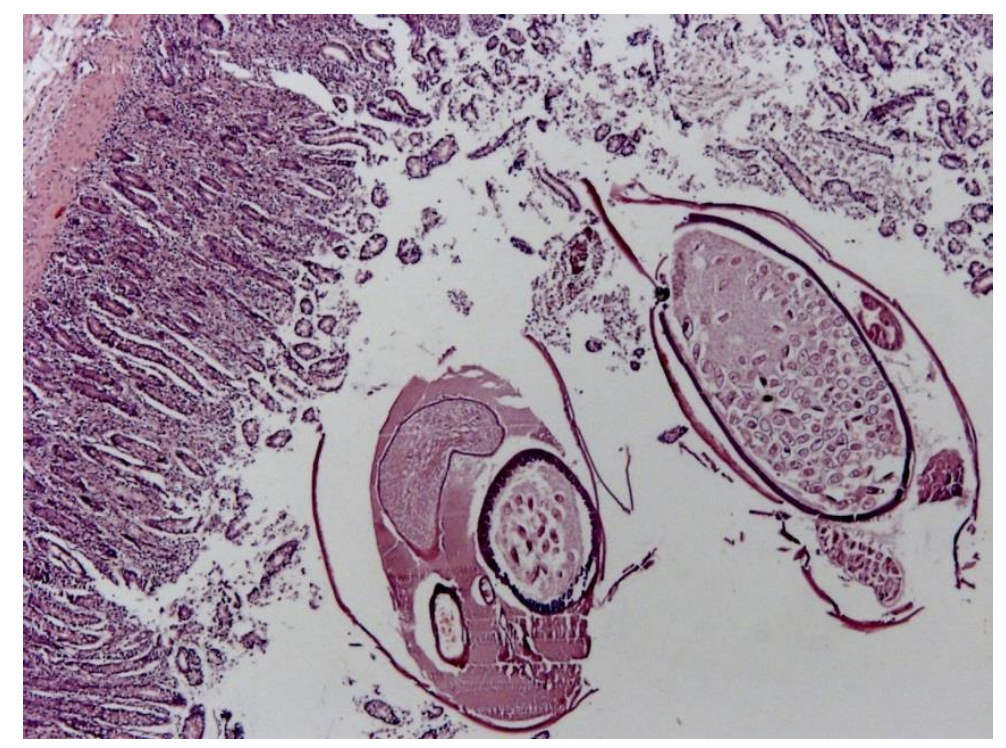

Figure 5. Caecum. Dystrophic changes in the mucosal epithelium layer. H/E, 10X

Based on the results we assume that $T$. ovis infection is accompanied by weak dystrophic changes in the mucosal epithelium (Figure 5) and inflammatory infiltrate consisting of macrophages, lymphocytes, neutrophils and single eosinophils. The investigations of
Kirkova et al. (8) on histopathology in $T$. vulpis infection in dogs have shown that massive colonization of the intestinal mucosa leads to extensive local inflammation which supports our results. We found that the cellular infiltration of macrophages and lymphocytes 
are mainly focused on areas around the parasite bodies. Surrounding mucosa was found to be infiltrated with lymphocytes and neutrophils. According to Bratanov et al. (6) a mild to moderate cell infiltration of the mucosa and submucosa by eosinophils, macrophages, lymphocytes and plasma cells have been found in $81.81 \%$ of sheep experimentally infected with T. ovis. At $18.18 \%$ of animals the eosinophils have not been found even around parasites body, but the presence of single or a greater number of fibroblasts has been observed. Our results also indicate that the basic cells in the inflammatory infiltrate are macrophages and lymphocytes (Figures 1 and 2a, b) but single eosinophils and fibroblasts were also discovered (Figure $\mathbf{2 b}$ ).

The experiments with sheep and rodents show that the acute inflammation plays a key role for expulsion of intestinal parasites. Using murine model Koyama et al. (11) and Else et al. (12) found that the immunity to $T$. muris infection leads to development of Th2 type immune response and it is wholly dependent on the presence of helper CD4+ $\mathrm{T}$ lymphocytes. Moreover, the development of inflammation is associated with the presence of macrophage accumulation in the affected areas (13). In our study the presence mainly of macrophages (CD68+) and CD4+ cells in the affected locations is indicative for developing protective inflammatory reaction. In contrast, eosinophils and mastocites are the two main Th2 effector cells associated with helminthic infections (14). Our results showed only single eosinophils in examined tissues. In the present study we did not differentiate mast cells because, in order to confirm the density of infiltration is necessary to use a mast cell tryptase or specific staining with toluidine blue.

\section{CONCLUSION}

The differences between presented reference and our results could be explained by the different comparison patterns used. For this reason, we believe that more additional studies should be conducted to fully clarify the cellular mechanism of protection against $T$. ovis infection in sheep.

\section{REFERENCES}

1. Kuchai, J., Ahmad, F., Chishti, M., Dar, J., Tak, H., On Morphology and Morphometry of Trichuris ovis Abildgaard, 1795 recovered from Ruminants of Ladakh, India. Journal of Buffalo Science, 2:49-52, 2013.

2. Singh, T. and Lal, S., A study on in vitro culture of Trichuris ovis in different physiological solutions at constant temperature, $37^{0}$ C. Journal of Parasitic Diseases, 35:57-60, 2011.

3. Soulsby, E., Helminths, Arthropods and Protozoa of domesticated animals. Sixth edition, Bailliere London, 318-321, 1968.

4. Liu, G., Wang, Y., Xu, M., Zhou, D., Ye, Y., Li, J., Song, H., Lin, R., Zhu, X., Characterization of the complete mitochondrial genomes of two whipworms Trichuris ovis and Trichuris discolor (Nematoda: Trichuridae). Infection, Genetics and Evolution, 8:1635-1641, 2012.

5. Beer, R., Morphological descriptions of the eggs and larval stages of Trichuris suis Schrank, 1799. Parasitology, 67:263-278, 1973.

6. Bratanov, V. and Enchev, S., Pathomorphological studies on trichocephalosis in sheep. Veterinary Science, 14:86-93, 1977.

7. Charakchiev, V., Studies on trichocephalosis in sheep. $\mathrm{PhD}$ thesis, Author's summery, 6-9, 1977.

8. Kirkova, Z. and Dinev, I., Morphological changes in the intestine of dogs, experimentally infected with Trichuris vulpis, Bulgarian Journal of Veterinary Medicine, 8:239-243, 2005.

9. Pittman, J., Shepherd, G., Thacker, B., Myers G., Trichuris suis in finishing pigs: Case report and review. Journal of Swine Health and Production, 18:306-313, 2010.

10.Smith, H. and Stevenson R., A clinical outbreak of Trichuris discolor infection in stabled calves. Canadian Veterinary Journal, 11:102-104, 1970.

11.Koyama K., Tamanchi H., Ito, Y., The role of $\mathrm{CD} 4+$ and $\mathrm{CD} 8+\mathrm{T}$ cells in protective immunity to

12.the murine parasite Trichuris muris. Parasite Immunology, 17:161-165, 1995.

13.Else, K. J. And Grencis, R. K., Antibodyindependent effector mechanisms in resistance to the intestinal nematode parasite Trichuris muris. Infection and Immunity, 64:2950-2954, 1996.

14. Matthew, L., Martinez-Pomares, L., Gordon, S., Else1, K., The mannose receptor binds Trichuris muris excretory/secretory proteins but is not essential for protective immunity. Immunology, 126:246-255, 2008.

15.Betts, C. and Else, K., Mast cells, eosinophils and antibody-mediated cellular cytotoxicity are not critical in resistance to Trichuris muris. Parasite Immunology, 21:45-52, 1999. 
ILIEV P.T., et al. 